\title{
Multiple photon Monte Carlo simulation for polarized Møller scattering with Yennie-Fraustchi-Suura exponentiation at high energies
}

\author{
S. Jadach \\ Institute of Nuclear Physics, Kraków, ul. Kawiory 26a, Poland \\ and CERN, Theory Division, CH-1211 Geneva 23, Switzerland \\ B. F. L. Ward \\ SLAC, P.O. Box 4349, Stanford University, Stanford, California 94309 \\ and Department of Physics and Astronomy, University of Tennessee, Knoxville, Tennessee 37996-1200
}

(Received 15 November 1995)

\begin{abstract}
We present the theoretical basis and sample Monte Carlo data for the YFS exponentiated $O(\alpha)$ calculation of polarized Møller scattering at c.m.s. energies large compared to $2 m_{e}$. Both longitudinal and transverse polarizations are discussed. Possible applications to Møller polarimetry at the SLD are thus illustrated. [S0556-2821(96)05713-X]

PACS number(s): 11.15.Tk, 12.15.Ji, 12.20.Ds, 13.10.+q
\end{abstract}

\section{INTRODUCTION}

The problem of high energy polarized $e^{-} e^{-} \rightarrow e^{-} e^{-}$scattering, Mbller scattering, is intimately connected with the analogous problem of high energy $e^{+} e^{-} \rightarrow e^{+} e^{-}$scattering, Bhabha scattering. Indeed, the two go into one another under standard crossing transformations. While the latter process has and continues to have substantial theoretical and experimental attention [1] due to its central role in the luminosity measurements at the CERN $e^{+} e^{-}$collider LEP and SLAC Linear Collider (SLC), the former process has traditionally only received moderate attention [2] due to a lack of an appropriate platform for detailed comparison between theory and experiment.

Recently, the situation has changed somewhat due to the success of the SLC Large Detector (SLD) Collaboration's measurement program for the famous left-right asymmetry $A_{L R}$ for polarized electrons and unpolarized positrons in the $e^{+} e^{-}$annihilation cross section in the $Z^{0}$ resonance region [3]. Indeed, one method of measuring the incident $e^{-}$polarization utilizes the polarization dependence of the Møller scattering cross section for polarized electrons in the SLC beam on a stationary polarized foil of known polarizationso-called Møller polarimetry. While this method of measurement is not the primary method of measuring the SLC $e^{-}$ polarization, it is used [4] as an important cross check on the more accurate Compton polarimetry employed by the SLD Collaboration in their high precision $A_{L R}$ studies. Accordingly, radiative corrections to polarized Møller scattering at the below $1 \%$ precision level are now of some interest. In this paper, we present the first results on the YennisFraustchi-Suura (YFS) [5] exponentiated exact $O(\alpha)$ Monte Carlo approach [6] to these corrections in analogy with the results on the crossed Bhabha process presented in $[7,8]$, for example.

Specifically, we extend the BHLUMI 1.xx Monte Carlo event generator in Ref. [7] to the crossed reaction of interest here. The extension involves introducing a new polarized matrix element into BHLUMI which is exact to $O(\alpha)$ for arbitrarily polarized incoming $e^{-}$beams. To this end, we follow the work of Ref. [9] for polarized Bhabha scattering and obtain the required exact $O(\alpha)$ matrix elements by crossing transformations on the virtual and soft corrections in this latter reference. For the corresponding $O(\alpha)$ hard bremsstrahlung matrix element for Møller scattering, we find it convenient to calculate it directly. These exact $O(\alpha)$ results are then combined with our standard YFS Monte Carlo methods as they pertain to Møller scattering (the respective YFS form factors are obtained via crossing from those already in BHLUMI 1.xx) to yield the first ever realistic multiple photon Monte Carlo event generator for Møller scattering with arbitrary initial beam polarizations. We call this new event generator BMOLLR.

In what follows, we first review the relevant aspects of BHLUMI 1.xx insofar as our crossing transformations to the Mbller process are concerned. This is done in the next section. In Sec. III, we present the respective crossed virtual and soft corrections derived from Ref. [9]. In Sec. IV, we calculate the desired hard bremsstrahlung correction. In Sec. V, we combine our results to create BMOLLR and we present sample Monte Carlo data to illustrate the corresponding multiple photon corrections. Comparisons with earlier analytic work in Refs. [10-12] as well as with recent SLD results [3] are also discussed in this section. Section VI contains some summary remarks.

\section{PRELIMINARIES}

In this section we review the relevant aspects of our YFS Monte Carlo methods as they pertain to the problem of extending our BHLUMI 1.xx event generator in Ref. [7] to the Møller process. In this way we also set our notation and define our kinematics.

More precisely, the problem we study herein is illustrated in Fig. 1, together with the respective kinematics: $e^{-}+e^{-} \rightarrow e^{-}+e^{-}+n(\gamma)$ at c.m.s. energies $\sqrt{s} \approx 0.22 \mathrm{GeV}$, so that we may work in the limit $m_{e}^{2} / s \ll 1$. This corresponds to the case of an SLC or LEP energy electron of energy 45.6 $\mathrm{GeV}$ incident upon an at rest electron in a polarized foil for example. In reality, the electrons in the foil would have some significant kinetic energy as well, so that we will allow the value of $s$ to vary in our eventual Monte Carlo simulation of 


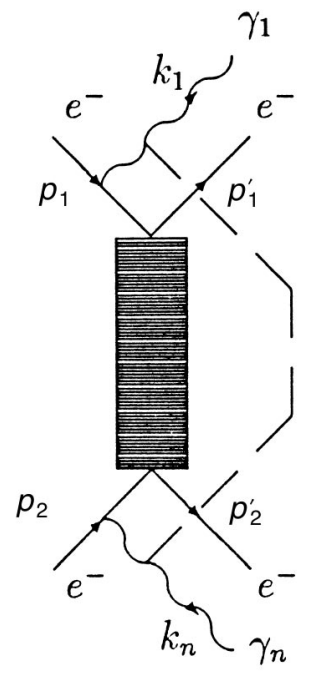

FIG. 1. The process $e^{-}+e^{-} \rightarrow e^{-}+e^{-}+n(\gamma)$. The fourmomenta are indicated in the standard manner: $p_{1}$ is the fourmomentum of the incoming $e^{-}, p_{2}^{\prime}$ is the four-momentum of one outgoing $e^{-}$, etc.

this Mфller process. In Ref. [7], we have realized by Monte Carlo methods the process $e^{+}+e^{-} \rightarrow e^{+} e^{-}+n(\gamma)$ via the fundamental YFS formula

$$
\begin{aligned}
d \sigma= & e^{2 \alpha \operatorname{Re} B+2 \alpha \widetilde{B}} \sum_{n=0}^{\infty} \frac{1}{n !} \int \prod_{j=1}^{n} \frac{d^{3} k_{j}}{k_{j}^{0}} \int \frac{d^{4} y}{(2 \pi)^{4}} \\
& \times \exp \left[i y\left(p_{1}+p_{2}-p_{1}^{\prime}-p_{2}^{\prime}-\sum_{j} k_{j}\right)+D\right] \\
& \times \bar{\beta}_{n}\left(k_{1}, \ldots, k_{n}\right) \frac{d^{3} p_{1}^{\prime} d^{3} p_{2}^{\prime}}{p_{1}^{\prime 0} p_{2}^{\prime 0}}
\end{aligned}
$$

where the real infrared function $\widetilde{B}$ and the virtual infrared function $B$ are given in Refs. $[5-8,13]$, and where we note the usual connections

$$
\begin{gathered}
2 \alpha \widetilde{B}=\int k \leqslant K_{\max } \frac{d^{3} k}{k_{0}} \widetilde{S}(k), \\
D=\int d^{3} k \frac{\widetilde{S}(k)}{k^{0}}\left[e^{-i y \cdot k}-\theta\left(K_{\max }-k\right)\right]
\end{gathered}
$$

for the standard YFS infrared emission factor

$$
\tilde{S}(k)=\frac{\alpha}{4 \pi^{2}}\left[Q_{f} Q_{f}^{(-)^{\prime}}\left(\frac{p_{1}}{p_{1} \cdot k}-\frac{p_{2}}{p_{2} \cdot k}\right)^{2}+\cdots\right]
$$

if $Q_{f}$ is the electric charge of $f$ in units of the position charge. Here, the ellipsis represents the remaining terms in $\widetilde{S}(k)$ obtained from the one given by respective substitutions of $Q_{f}, p_{1}, Q_{f}^{(-)^{\prime}}, p_{2}$ with corresponding values for the other pairs of the external fermion legs in Fig. 1 according to the YFS prescription in Ref. [5] (wherein due attention is taken to obtain the correct relative sign of each of the terms in $\widetilde{S}(k)$ according to this latter prescription) and in Ref. [7], $f=e$, $\stackrel{(-)^{\prime}}{f}=\bar{e}$.
The YFS hard photon residuals $\bar{\beta}_{i}$ in (1), $i=0,1$, are given in Ref. [7] for BHLUMI 1.xx so that this latter event generator calculates the YFS exponentiated exact $O(\alpha)$ cross section for Bhabha scattering using a corresponding Monte Carlo realization of (1). In the next sections, we use crossing and explicit Feynman diagrammatic methods to extend the realization of (1) in BHLUMI 1.xx to the corresponding Monte Carlo realization of the respective application of (1) to Møller scattering.

\section{SOFT AND VIRTUAL CORRECTIONS AT $O(\alpha)$}

In this section, we present the required formulas for extending the exact $O(\alpha)$ Monte Carlo realization of the hard photon residual $\bar{\beta}_{0}$ in BHLUMI 1.Xx [7] to the corresponding Monte Carlo realization for the Møller process. We begin by recording the respective Born differential cross section.

Referring to the kinematics in Fig. 1, we define spin fourvectors for the incident (beam) and target (foil) electrons in the corresponding incoming rest frames as

$$
\begin{aligned}
& s_{1}=\left(0, P_{1 t} \hat{s}_{1}\left(\phi_{1}\right), P_{1 z} \hat{z}\right), \\
& s_{2}=\left(0, P_{2 t} \hat{s}_{2}\left(\phi_{2}\right), P_{2 z} \hat{z}\right),
\end{aligned}
$$

where

$$
\hat{s}_{i}=\cos \phi_{i} \hat{x}+\sin \phi_{i} \hat{y}
$$

and $P_{i h}, i=1,2, h=t, z$ are the polarizations of the incident beam $(i=2)$ and target $(i=1)$ electrons along the transverse $(h=t)$ and longitudinal $(h=z)$ directions when the incoming beam direction is taken as the $z$ axis for definiteness in the usual $x-y-z$ Cartesian coordinate system for the c.m.s. system. Here, $\hat{a}, a=x, y, z$ is a unit vector in the $a$ direction as usual and the angles $\phi_{i}$ are arbitrary. Accordingly, (4) and (5) represent an arbitrary incoming polarization state for the Møller process.

The respective Born differential cross section associated with the incoming state represented by (4) and (5) is well known and can be obtained by the standard manipulations on the Born amplitude associated with Fig. 1. We get the result

$$
d \sigma_{\mathrm{Born}} / d \Omega=F_{\mathrm{pol}} d \bar{\sigma}_{\mathrm{Born}} / d \Omega
$$

for

$$
d \bar{\sigma}_{\text {Born }} / d \Omega=\frac{\alpha^{2}}{2 s}\left(\frac{s^{2}+t^{2}}{u^{2}}+\frac{s^{2}+u^{2}}{t^{2}}+\frac{2 s^{2}}{u t}\right)
$$

and

$$
F_{\mathrm{pol}}=1-P_{1 z} P_{2 z} A_{z}-P_{1 t} P_{2 t} A_{t} \cos \left(\phi_{1}+\phi_{2}-2 \varphi_{2 \mathrm{c} . \mathrm{m} .}^{\prime}\right)
$$

with

$$
\begin{gathered}
A_{z}=1-\left\{t^{2} / u^{2}+u^{2} / t^{2}\right\} /\left\{\frac{1}{2}\left(\frac{s^{2}+t^{2}}{u^{2}}+\frac{s^{2}+u^{2}}{t^{2}}+\frac{2 s^{2}}{u t}\right)\right\} \\
A_{t}=1 /\left\{\frac{1}{2}\left(\frac{s^{2}+t^{2}}{u^{2}}+\frac{s^{2}+u^{2}}{t^{2}}+\frac{2 s^{2}}{u t}\right)\right\}
\end{gathered}
$$


Here, the angle $\varphi_{2 \text { c.m. }}^{\prime}$ is the c.m. system (c.m.s.) azimuthal scattering angle of the final electron 4-momentum $p_{2}^{\prime}$ in Fig. 1. Here, we have defined the Mandelstam invariants

$$
s=\left(p_{1}+p_{2}\right)^{2}, \quad t=\left(p_{1}-p_{1}^{\prime}\right)^{2}, \quad u=\left(p_{1}-p_{2}^{\prime}\right)^{2},
$$

and we note that it is sometimes convenient to represent the $A_{h}, h=z, t$ as

$$
\begin{gathered}
A_{z}=\left(7+\cos ^{2} \theta_{\text {c.m. }}\right) \sin ^{2} \theta_{\text {c.m. }} /\left(3+\cos ^{2} \theta_{\text {c.m. }}\right)^{2} \\
A_{t}=\sin ^{4} \theta_{\text {c.m. }} /\left(3+\cos ^{2} \theta_{\text {c.m. }}\right)^{2},
\end{gathered}
$$

where $\theta_{\text {c.m. }}$ is the corresponding c.m.s. polar scattering angle. It is this Born distribution which we must introduce into (1) and realize via our Monte Carlo methods in complete analogy with our analysis for Bhabha scattering in Ref. [7]. Here, we may note that this may be done by introducing the factor $F_{\text {pol }}$ into the Born distribution, the $O\left(\alpha^{0}\right)$ contribution to $\bar{\beta}_{0}$, in BHLUMI 1.xx in Ref. [7] after crossing that distribution to the Møller process. We have done this.

We may now turn to the corresponding $O(\alpha)$ corrections for the hard photon residuals $\bar{\beta}_{i}, i=0,1$ for the Mbller process. We start by discussing soft and virtual $O(\alpha)$ corrections in the remainder of this section; the respective hard bremsstrahlung correction is then presented in the following section.

As we implied above, in the YFS theory, there are already published explicit results in Refs. [5-8,13] for the real emission and virtual infrared functions $\widetilde{B}$ and $B$ in the processes $e^{+}+e^{-} \rightarrow f+\bar{f}$, where $f$ is a fermion of charge $Q_{f}$ in units of the positron charge. These results are realized explicitly for Bhabha scattering in the Ref. [7]. Thus, here, we simply perform crossing on the results for $\widetilde{B}$ and $B$ in BHLUMI 1.xx in Ref. [7] to obtain the analogous function for the Møller process. This completes our discussion of the soft real emission and soft virtual corrections for Mфller scattering.

Turning now to the hard virtual corrections, we refer to the corresponding results for the Bhabha process in Ref. [9]. In this latter reference, the $O(\alpha)$ QED virtual corrections to Bhabha scattering are presented in a helicity amplitude basis so that required polarization information is available upon crossing these amplitudes to the M $\phi$ ller process. Proceeding in this way, we get the result

$$
\begin{aligned}
\frac{1}{2} \bar{\beta}_{0}^{1 \text { loop }}= & \frac{d \sigma_{\text {Born }}}{d \Omega}\left[1+\delta_{\text {unpol }}-2 \alpha(\operatorname{Re} B+\widetilde{B})+\frac{P_{1 z} P_{2 z}}{F_{\text {pol }}} \frac{\left(t^{2} / u^{2}\right)\left(\delta_{\| 2}-\delta_{\text {unpol }}\right)+\left(u^{2} / t^{2}\right)\left(\delta_{\| 3}-\delta_{\text {unpol }}\right)}{\frac{s}{\alpha^{2}} d \bar{\sigma}_{\text {Born }} / d \Omega}\right. \\
& \left.-\frac{P_{1 t} P_{2 t}}{F_{\text {pol }}} A_{t} \cos \left(\phi_{1}+\phi_{2}-2 \varphi_{2 \text { c.m. }}^{\prime}\right)\left(\delta_{\perp}-\delta_{\text {unpol }}\right)\right],
\end{aligned}
$$

where $\delta_{\perp}=\left(\delta_{\mid 2}+\delta_{\mid 3}\right) / 2, \delta_{\mid 2}, \delta_{\mid 3}$ are obtained by crossing from the corresponding corrections $\delta_{2}, \delta_{3}$, respectively, for Bhabha scattering given in Ref. [9] for the helicity amplitudes $M_{2}, M_{3}$ as defined therein, $\delta_{\text {unpol }}$ denotes the unpolarized soft + virtual correction for Bhabha scattering given in Refs. [7, 9] crossed into the Møller channel, and $d \bar{\sigma}_{\text {Born }} d \Omega$ is the unpolarized Møller differential cross section at the Born level given in (7). The superscript 1 loop on $\bar{\beta}_{0}$ indicates that it is computed through one-loop order.

This completes our discussion of the soft and virtual corrections for polarized Møller scattering at high energies. We turn next to the hard $O(\alpha)$ bremsstrahlung correction in the following section.

\section{HARD BREMSSTRAHLUNG AT $O(\alpha)$}

In this section we complete our construction of the hard photon residuals needed for our realization of the $O(\alpha)$ YFS exponentiated cross section for polarized Møller scattering by computing the respective $O(\alpha)$ bremsstrahlung correction. We begin by setting our calculational notation.

Specifically, we shall use the helicity amplitude methods pioneered by the CALKUL Collaboration [14] as they were realized by Xu et al. in Ref. [15]. Kleiss and Stirling [16] have developed an equivalent realization. This means that we write our external fermion wave functions as $\left\langle q h|,| q^{\prime} h^{\prime}\right\rangle$ for outgoing and incoming states with four-momentum $q$ and helicity $h$ and with four-momentum $q^{\prime}$ and helicity $h^{\prime}$, respectively, $h, h^{\prime}=+,-$. The photon polarization vectors of helicity $h=+(-)$ for a photon with four-momentum $k$ are then

$$
\epsilon_{\mu}^{*}(k)_{h}=\frac{\left\langle p-h\left|\gamma_{\mu}\right| k-h\right\rangle}{\sqrt{2}\langle p \mid k\rangle^{(*)}}
$$

where we have defined the spinor product as

$$
\langle p \mid k\rangle \equiv\langle p-\mid k+\rangle,
$$

for

$$
\langle p-\mid k+\rangle=\frac{\left(p_{x}+i p_{y}\right) \sqrt{k_{+}}}{\sqrt{p_{+}}}-\frac{\sqrt{p_{+}}\left(k_{x}+i k_{y}\right)}{\sqrt{k_{+}}},
$$

where we require $p^{2}=0$. Here, $v_{+}=v^{0}+v_{z}, v_{a}=\vec{v} \cdot \hat{a}$, $a=x, y, z$, for all four-vectors $v$; here, our metric on Minkowski space is such that $v^{\mu}=\left(v^{0}, v_{x}, v_{y}, v_{z}\right)$. As usual, the auxilliary four-vector $p$ may be chosen to simplify the respective amplitudes according to the pioneering methods in Refs. $[14,15]$. We need to stress that, due to the required 
phase information for the transverse spin effects, we were unable to use the results in Ref. [17], which under other circumstances could have been used together with crossing to obtain the required fully polarized $O(\alpha)$ bremsstrahlung correction to Møller scattering.

With these further notational conventions explicitly recorded, let us first note that, since the unpolarized $O(\alpha)$ bremsstrahlung cross section for Møller scattering, $d \sigma^{B 1} /\left.d \Omega k d k d \Omega_{\gamma}\right|_{\text {unpol }}$, is available via crossing from the corresponding unpolarized $O(\alpha)$ bremsstrahlung correction to Bhabha scattering in Ref. [7], what we need to calculate here is really the ratio of the polarized and unpolarized $O(\alpha)$ bremsstrahlung cross sections for the Møller process. This will then allow us to compute $\bar{\beta}_{1}$ in (1) by using this ratio, $d \sigma^{B 1} / d \sigma^{B} \overline{1}_{\text {unpol }}$, via the standard YFS formula

$$
\begin{aligned}
\frac{1}{2} \bar{\beta}_{1}= & \left\{d \sigma^{B 1} /\left.d \sigma^{B 1}\right|_{\text {unpoll }}\left\{d \sigma^{B 1} /\left.\left(d \Omega k d k d \Omega_{\gamma}\right)\right|_{\text {unpol }}\right\}\right. \\
& -\widetilde{S}(k)\left\{d \sigma_{\text {Born }} / d \Omega\right\} .
\end{aligned}
$$

Using the identification $\mathscr{C}\left(h_{1} h_{2} h_{1}^{\prime} h_{2}^{\prime} h_{\gamma}\right)$ for the respective Feynman amplitude for the incoming $e^{-}$with fourmomentum $p_{1}$ and helicity $h_{1}$ and the incoming $e^{-}$with four-momentum $p_{2}$ and helicity $h_{2}$ to scatter to the final state in which the outgoing $e^{-}$with four-momentum $p_{i}^{\prime}$ has helicity $h_{i}^{\prime}, i=1,2$, and in which the outgoing photon has fourmomentum $k$ and helicity $h_{\gamma}$, as we illustrate diagrammatically as the case $n=1$ in Fig. 1 , we find the basic result

$$
\begin{aligned}
& d \sigma^{B 1} /\left.d \sigma^{B 1}\right|_{\text {unpol }}=1-P_{1 z} P_{2 z}\left(1-\frac{\frac{1}{2} \sum_{h_{i}^{\prime}, h_{\gamma}}\left(\left|\mathscr{L C}\left(-+h_{1}^{\prime} h_{2}^{\prime} h_{\gamma}\right)\right|^{2}+\left|\mathscr{L}\left(+-h_{1}^{\prime} h_{2}^{\prime} h_{\gamma}\right)\right|^{2}\right)}{\frac{1}{4} \sum_{h_{i}, h_{i}^{\prime}, h_{\gamma}}\left|\mathscr{L}\left(h_{1} h_{2} h_{1}^{\prime} h_{2}^{\prime} h_{\gamma}\right)\right|^{2}}\right) \\
& +\frac{P_{1 t} P_{2 t}}{2} \frac{\operatorname{Re} \sum_{h_{i}^{\prime}, h_{\gamma}} \mathscr{L l}^{*}\left(+-h_{1}^{\prime} h_{2}^{\prime} h_{\gamma}\right) \mathscr{L C}\left(-+h_{1}^{\prime} h_{2}^{\prime} h_{\gamma}\right) e^{i\left(\phi_{1}+\phi_{2}\right)}}{\frac{1}{4} \sum_{h_{i}, h_{i}^{\prime}, h_{\gamma}}\left|\mathscr{L}\left(h_{1} h_{2} h_{1}^{\prime} h_{2}^{\prime} h_{\gamma}\right)\right|^{2}} .
\end{aligned}
$$

Accordingly, our required formulas for completing our YFS $O(\alpha)$ exponentiated polarized Mфller scattering calculation will be complete when we list the formulas for the 12 independent nonzero amplitudes $\mathscr{L}\left(h_{1} h_{2} h_{1}^{\prime} h_{2}^{\prime} h_{\gamma}\right)$. We find the results

$$
\begin{aligned}
& \mathscr{L C}(+++++)=-2 i \sqrt{2} e_{0}^{3} s_{0} \frac{\left\langle p_{2} \mid p_{1}\right\rangle}{\left\langle p_{2} \mid p_{1}\right\rangle^{*}}\left(\frac{1}{t_{1}} \frac{\left\langle p_{1}+\mid p_{1}^{\prime}-\right\rangle}{\left\langle p_{2} \mid k\right\rangle\left\langle k \mid p_{2}^{\prime}\right\rangle}+\frac{1}{t_{0}} \frac{\left\langle p_{2}+\mid p_{2}^{\prime}-\right\rangle}{\left\langle p_{1} \mid k\right\rangle\left\langle k \mid p_{1}^{\prime}\right\rangle}-\frac{1}{u_{1}} \frac{\left\langle p_{1}+\mid p_{2}^{\prime}-\right\rangle}{\left\langle p_{2} \mid k\right\rangle\left\langle k \mid p_{1}^{\prime}\right\rangle}-\frac{1}{u_{0}} \frac{\left\langle p_{2}+\mid p_{1}^{\prime}-\right\rangle}{\left\langle p_{1} \mid k\right\rangle\left\langle k \mid p_{2}^{\prime}\right\rangle}\right), \\
& \mathscr{L C}(++++-)=2 i \sqrt{2} e_{0}^{3} s_{1} \frac{\left\langle p_{1}^{\prime} \mid p_{2}^{\prime}\right\rangle^{*}}{\left\langle p_{1}^{\prime} \mid p_{2}^{\prime}\right\rangle}\left(\frac{1}{t_{1}} \frac{-\left\langle p_{1}^{\prime}-\mid p_{1}+\right\rangle}{\left\langle k \mid p_{2}\right\rangle^{*}\left\langle p_{2}^{\prime} \mid k\right\rangle^{*}}+\frac{1}{t_{0}} \frac{-\left\langle p_{2}^{\prime}-\mid p_{2}+\right\rangle}{\left\langle p_{1}^{\prime} \mid k\right\rangle^{*}\left\langle k \mid p_{1}\right\rangle^{*}}-\frac{1}{u_{1}} \frac{-\left\langle p_{2}^{\prime}-\mid p_{1}+\right\rangle}{\left\langle k \mid p_{2}\right\rangle^{*}\left\langle p_{1}^{\prime} \mid k\right\rangle^{*}}\right. \\
& \left.-\frac{1}{u_{0}} \frac{-\left\langle p_{1}^{\prime}-\mid p_{2}+\right\rangle}{\left\langle k \mid p_{1}\right\rangle^{*}\left\langle p_{2}^{\prime} \mid k\right\rangle^{*}}\right) \\
& \mathscr{b}(----+)=-2 i \sqrt{2} e_{0}^{3} s_{1} \frac{\left\langle p_{2}^{\prime} \mid p_{1}^{\prime}\right\rangle}{\left\langle p_{2}^{\prime} \mid p_{1}^{\prime}\right\rangle^{*}}\left(\frac{1}{t_{1}} \frac{\left\langle p_{1}+\mid p_{1}^{\prime}-\right\rangle}{\left\langle k \mid p_{2}\right\rangle\left\langle p_{2}^{\prime} \mid k\right\rangle}+\frac{1}{t_{0}} \frac{\left\langle p_{2}+\mid p_{2}^{\prime}-\right\rangle}{\left\langle k \mid p_{1}\right\rangle\left\langle p_{1}^{\prime} \mid k\right\rangle}-\frac{1}{u_{1}} \frac{\left\langle p_{1}+\mid p_{2}^{\prime}-\right\rangle}{\left\langle k \mid p_{2}\right\rangle\left\langle p_{1}^{\prime} \mid k\right\rangle}-\frac{1}{u_{0}} \frac{\left\langle p_{2}+\mid p_{1}^{\prime}-\right\rangle}{\left\langle k \mid p_{1}\right\rangle\left\langle p_{2}^{\prime} \mid k\right\rangle}\right), \\
& \mathscr{C l}(-----)=-2 i \sqrt{2} e_{0}^{3} s_{0} \frac{\left\langle p_{1} \mid p_{2}\right\rangle^{*}}{\left\langle p_{1} \mid p_{2}\right\rangle}\left(\frac{1}{t_{1}} \frac{-\left\langle p_{1}-\mid p_{1}^{\prime}+\right\rangle}{\left\langle p_{2} \mid k\right\rangle^{*}\left\langle k \mid p_{2}^{\prime}\right\rangle^{*}}+\frac{1}{t_{0}} \frac{-\left\langle p_{2}-\mid p_{2}^{\prime}+\right\rangle}{\left\langle k \mid p_{1}^{\prime}\right\rangle^{*}\left\langle p_{1} \mid k\right\rangle^{*}}-\frac{1}{u_{1}} \frac{-\left\langle p_{1}-\mid p_{2}^{\prime}+\right\rangle}{\left\langle k \mid p_{1}^{\prime}\right\rangle^{*}\left\langle p_{2} \mid k\right\rangle^{*}}\right. \\
& \left.-\frac{1}{u_{0}} \frac{-\left\langle p_{2}-\mid p_{1}^{\prime}+\right\rangle}{\left\langle k \mid p_{2}^{\prime}\right\rangle^{*}\left\langle p_{1} \mid k\right\rangle^{*}}\right) \\
& \mathscr{L C}(+-+--)=2 i \sqrt{2} e_{0}^{3} u_{0} \frac{\left\langle p_{1}^{\prime} \mid p_{2}\right\rangle^{*}}{\left\langle p_{1}^{\prime} \mid p_{2}\right\rangle}\left(\frac{1}{t_{1}} \frac{\left\langle p_{1}^{\prime}-\mid p_{1}+\right\rangle}{\left\langle k \mid p_{2}^{\prime}\right\rangle^{*}\left\langle p_{2} \mid k\right\rangle^{*}}+\frac{1}{t_{0}} \frac{\left\langle p_{2}^{\prime}-\mid p_{2}+\right\rangle}{\left\langle k \mid p_{1}^{\prime}\right\rangle^{*}\left\langle p_{1} \mid k\right\rangle^{*}}\right), \\
& \mathscr{L}(+-+-+)=-2 i \sqrt{2} e_{0}^{3} u_{1} \frac{\left\langle p_{2}^{\prime} \mid p_{1}\right\rangle}{\left\langle p_{2}^{\prime} \mid p_{1}\right\rangle^{*}}\left(\frac{1}{t_{1}} \frac{\left\langle p_{1}^{\prime}+\mid p_{1}-\right\rangle}{\left\langle k \mid p_{2}^{\prime}\right\rangle\left\langle p_{2} \mid k\right\rangle}+\frac{1}{t_{0}} \frac{\left\langle p_{2}^{\prime}+\mid p_{2}-\right\rangle}{\left\langle k \mid p_{1}^{\prime}\right\rangle\left\langle p_{1} \mid k\right\rangle}\right), \\
& \mathscr{C l}(-+-+-)=2 i \sqrt{2} e_{0}^{3} u_{1} \frac{\left\langle p_{2}^{\prime}+\mid p_{1}-\right\rangle}{\left\langle p_{2}^{\prime}+\mid p_{1}-\right\rangle^{*}}\left(\frac{1}{t_{1}} \frac{\left\langle p_{1}^{\prime}-\mid p_{1}+\right\rangle}{\left\langle k \mid p_{2}\right\rangle^{*}\left\langle p_{2}^{\prime} \mid k\right\rangle^{*}}+\frac{1}{t_{0}} \frac{\left\langle p_{2}^{\prime}-\mid p_{2}+\right\rangle}{\left\langle p_{1} \mid k\right\rangle^{*}\left\langle k \mid p_{1}^{\prime}\right\rangle^{*}}\right),
\end{aligned}
$$




$$
\begin{gathered}
\mathscr{L}(-+-++)=-2 i \sqrt{2} e_{0}^{3} u_{0} \frac{\left\langle p_{1}^{\prime} \mid p_{2}\right\rangle}{\left\langle p_{1}^{\prime} \mid p_{2}\right\rangle^{*}}\left(\frac{1}{t_{1}} \frac{\left\langle p_{1}^{\prime}+\mid p_{1}-\right\rangle}{\left\langle p_{2} \mid k\right\rangle\left\langle k \mid p_{2}^{\prime}\right\rangle}+\frac{1}{t_{0}} \frac{\left\langle p_{2}^{\prime}+\mid p_{2}-\right\rangle}{\left\langle k \mid p_{1}\right\rangle\left\langle p_{1}^{\prime} \mid k\right\rangle}\right), \\
\mathscr{L}(-++--)=2 i \sqrt{2} e_{0}^{3} t_{1} \frac{\left\langle p_{1} \mid p_{1}^{\prime}\right\rangle^{*}}{\left\langle p_{1} \mid p_{1}^{\prime}\right\rangle}\left(\frac{1}{u_{0}} \frac{-\left\langle p_{1}^{\prime}-\mid p_{2}+\right\rangle}{\left\langle p_{1} \mid k\right\rangle^{*}\left\langle k \mid p_{2}^{\prime}\right\rangle^{*}}+\frac{1}{u_{1}} \frac{-\left\langle p_{2}^{\prime}-\mid p_{1}+\right\rangle}{\left\langle p_{1}^{\prime} \mid k\right\rangle^{*}\left\langle k \mid p_{2}\right\rangle^{*}}\right), \\
\mathscr{L}(-++-+)=2 i \sqrt{2} e_{0}^{3} t_{0} \frac{\left\langle p_{2}^{\prime} \mid p_{2}\right\rangle}{\left\langle p_{2}^{\prime} \mid p_{2}\right\rangle^{*}}\left(\frac{1}{u_{0}} \frac{\left\langle p_{1}^{\prime}+\mid p_{2}-\right\rangle}{\left\langle k \mid p_{1}\right\rangle\left\langle p_{2}^{\prime} \mid k\right\rangle}+\frac{1}{u_{1}} \frac{\left\langle p_{2}^{\prime}+\mid p_{1}-\right\rangle}{\left\langle p_{2} \mid k\right\rangle\left\langle k \mid p_{1}^{\prime}\right\rangle}\right), \\
\mathscr{M}(+--+-)=-2 i \sqrt{2} e_{0}^{3} t_{0} \frac{\left\langle p_{2} \mid p_{2}^{\prime}\right\rangle^{*}}{\left\langle p_{2} \mid p_{2}^{\prime}\right\rangle}\left(\frac{1}{u_{0}} \frac{\left\langle p_{1}^{\prime}-\mid p_{2}+\right\rangle}{\left\langle k \mid p_{1}\right\rangle^{*}\left\langle p_{2}^{\prime} \mid k\right\rangle^{*}}+\frac{1}{u_{1}} \frac{\left\langle p_{2}^{\prime}-\mid p_{1}+\right\rangle}{\left\langle p_{2} \mid k\right\rangle^{*}\left\langle k \mid p_{1}^{\prime}\right\rangle^{*}}\right), \\
\mathscr{M}(+--++)=2 i \sqrt{2} e_{0}^{3} t_{1} \frac{\left\langle p_{1} \mid p_{1}^{\prime}\right\rangle}{\left\langle p_{1} \mid p_{1}^{\prime}\right\rangle^{*}}\left(\frac{1}{u_{0}} \frac{\left\langle p_{1}^{\prime}+\mid p_{2}-\right\rangle}{\left\langle p_{1} \mid k\right\rangle\left\langle k \mid p_{2}^{\prime}\right\rangle}+\frac{1}{u_{1}} \frac{\left\langle p_{2}^{\prime}+\mid p_{1}-\right\rangle}{\left\langle k \mid p_{2}\right\rangle\left\langle p_{1}^{\prime} \mid k\right\rangle}\right),
\end{gathered}
$$

where we have defined the invariants

$$
\begin{array}{lll}
s_{0}=\left(p_{1}+p_{2}\right)^{2}, & t_{0}=\left(p_{2}-p_{2}^{\prime}\right)^{2}, & u_{0}=\left(p_{2}-p_{1}^{\prime}\right)^{2}, \\
s_{1}=\left(p_{1}^{\prime}+p_{2}^{\prime}\right)^{2}, & t_{1}=\left(p_{1}-p_{1}^{\prime}\right)^{2}, & u_{1}=\left(p_{1}-p_{2}^{\prime}\right)^{2}
\end{array}
$$

in a standard manner. These amplitudes for the polarized hard bremsstrahlung effect in Mbller scattering then complete our specification of the formulas needed to calculate the hard photon residual $\bar{\beta}_{1}$ in (1). In this way, we have extended the $O(\alpha)$ YFS exponentiated Monte Carlo BHLUMI 1.xx in Ref. [7] to our $O(\alpha)$ YFS exponentiated Monte Carlo calculation of polarized Møller scattering at high energies. We call the respective Monte Carlo event generator BMOLLR 1.0 and it may be obtained from the authors upon request. In the next section, we illustrate it with some explicit Monte Carlo data in the kinematical regime relevant to the SLD measurement of their polarization via their Mbller polarimeter.

Before we end this section, we should like to point-out that the polarized hard bremsstrahlung results for M $\phi$ ller scattering presented in this section are the Mbller analoga of the corresponding polarized hard bremsstrahlung results for Bhabha scattering given by Kleiss in Ref. [18]. Indeed, we have checked that Kleiss' prescriptions in Ref. [18], when applied to Mфller scattering, yield results entirely equivalent to our formulas in this section.

\section{RESULTS}

In this section we illustrate various radiatively corrected polarized Møller scattering effects in the regime relevant for the SLD Møller polarimeter measurements. We begin with a brief review of the specific kinematics of interest.

More precisely, for the purposes of our illustration, the electron in the stationary foil will be taken to be at rest [4] in the laboratory frame, with the beam electron travelling along the $z$ axis with energy $45.6 \mathrm{GeV}$. The acceptance of the SLD Møller polarimeter will be taken as

$$
6 \mathrm{mrad} \leqslant \theta_{\text {lab }} \leqslant 9 \mathrm{mrad} \text {, }
$$

where $\theta_{\text {lab }}$ is the respective laboratory scattering angle. For simplicity we take the polarimeter detector to be azimuthally symmetric. The incoming longitudinal beam polarization, $P_{2 z}$, will be taken at the 1993 value of 0.62 with the foil polarization set at $P_{1 z}=1$ for definiteness-recently, higher beam polarizations, 0.8 , have been attained (see Swartz et al. in Ref. [2] for some discussion of the use of BMOLLR in the analysis of the SLD M $b$ ller polarimeter data). We emphasize that the polarization of any one beam enters the cross sections under discussion only linearly so that one may scale the respective effects in a straightforward way to other values of $P_{2 z}$. Finally, for completeness, we also discuss the input transverse polarizations

$$
P_{2 t}=0.62, \quad P_{1 t}=1, \quad P_{i z}=0, \quad \phi_{i}=\pi / 4, i=1,2 .
$$

Such configurations are of possible interest in checking for a possible rotation of the originally longitudinal polarization into the transverse spin space [4]. Here, we want to illustrate the size of the radiative multiple photon effects in both the transverse and longitudinal aspects of the beam polarization space.

We summarize our Monte Carlo data in Table I, where we have simulated $6 \times 10^{7}$ events per entry in this table.

What we see is that, for the longitudinal polarization, the presence of the polarization changes the radiative correction by $0.090 \pm 0.037 \%$ from its unpolarized value of -9.850 $\pm 0.027 \%$, whereas, for the transverse polarization, this change is $-0.071 \pm 0.047 \%$, so that it is below the $2 \sigma$ statistical error on our simulations. We need to stress that both of these effects are below the total precision $0.7 \%[7,19]$ on the simulations. From Refs. [10-12], we see that the overall size of the radiative corrections to the SLD acceptance is generally consistent with the size of the effects discussed therein. We cannot make a direct comparison with these references because their authors did not evaluate the hard bremsstrahlung effect numerically. However, we have checked that our Born cross sections agree with those given in Table I of Ref. [11] to the accuracy of this table and that our soft plus virtual corrections agree with the results in Table I of Ref. [10] at the expected accuracy of $0.1 \%$ at $O(\alpha)$, in view of the $0.1 \%$ accuracy of the numerical results presented in that table.

We can further conclude that, when issues of accuracy better than $10 \%$ are at issue in Moller polarimetry in the 
TABLE I. $O(\alpha)$ exponentiated results for the polarized M $\phi l l e r$ scattering into the SLD M $\phi l l e r$ polarimeter acceptance at the SLC where the incoming $e^{-}$beam has energy $45.6 \mathrm{GeV}$ and is incident on a stationary target foil. The Born results are shown for comparison. The total cross sections in nanobarns are denoted by $\sigma_{\text {tot }}(\mathrm{nb})$ whereas the analogous cross sections in nanobarns into the polarimeter acceptance are denoted by $\sigma_{\text {trig }}(\mathrm{nb})$. The transverse polarization results for $\sigma_{\text {trig }}(\mathrm{nb})$ are integrated only over $[0, \pi / 2]$ and $[\pi, 3 \pi / 2]$ in $\phi_{2 \mathrm{~cm}}^{\prime}$ to produce a nonzero polarization effect. The errors indicated are purely statistical. From Refs. [7,19], the total precision tag of the simulations is $\sim 0.7 \%$.

\begin{tabular}{lccc}
\hline \hline & & BMOLLR 1.0 \\
& $P_{i z}=P_{i t}=0$ & $P_{1 z}=1, P_{2 z}=0.62, P_{i t}=0$ & $P_{1 z}=0, P_{1 t}=1, P_{2 t}=0.62, \phi_{i}=\pi / 4$ \\
\hline$O(\alpha)^{\exp }$ & & & \\
$2 ! \sigma_{\text {tot }}(\mathrm{nb})$ & $12837.74 \pm 0.60$ & $8003.31 \pm 0.30$ & $12837.74 \pm 0.60$ \\
$2 ! \sigma_{\text {trig }}(\mathrm{nb})$ & $4634.22 \pm 1.00$ & $3064.02 \pm 0.64$ & $2260.74 \pm 0.70$ \\
Born & & & \\
$2 ! \sigma_{\text {tot }}(\mathrm{nb})$ & $13496.752 \pm 0.016$ & $8261.654 \pm 0.026$ & $13496.758 \pm 0.035$ \\
$2 ! \sigma_{\text {trig }}(\mathrm{nb})$ & $5140.57 \pm 1.08$ & $3395.41 \pm 0.68$ & $2509.74 \pm 0.75$ \\
\hline \hline
\end{tabular}

SLD-type kinematical regime, the radiative corrections must be computed to the desired accuracy, as they enter at the level of $\sim 10 \%$ in the respective cross sections.

\section{CONCLUSIONS}

In this paper we have extended our BHLUMI 1.xx Monte Carlo event generator to the polarized Møller scattering process at high energies with an eye toward the SLD M $\phi$ ller polarimeter. The result of this extension is the new $O(\alpha)$ YFS exponentiated Monte Carlo event generator BMOLLR and it is available from the authors upon request.

Specifically, for the process $e^{-} e^{-} \rightarrow e^{-} e^{-}+n(\gamma)$, BMOLLR provides an event-by-event simulation in which the final photon four-vectors are available together with the final charged particle four-vectors, so that each event may be subjected to arbitrary detector cuts as needed. The incoming beams may be arbitrarily polarized so that the interplay between polarization and radiation may be investigated in detail. We have illustrated such interplay and the size of the radiative effects in the SLD Møller polarimeter acceptance with sample Monte Carlo data. We find that longitudinal polarization modulates the size of the radiative corrections more than does transverse polarization but that both modu- lations are small and significantly less than the $0.7 \%$ total precision of the calculations. The size of the radiative effects, $\sim 10 \%$, necessitates that they be computed with good precision in any discussion of Mbller polarimetry with a precision tag better than $10 \%$. For the current SLD application [4], our $0.7 \%$ precision tag is adequate. A higher physical precision could be obtained if needed via methods analogous to what was done in arriving at version 2.xx of BHLUMI in Refs. [8], for example.

In summary, we have provided the first ever multiple photon Monte Carlo event generator with exact $O(\alpha)$ YFS exponentiation for polarized M $\phi l$ ler scattering at high energies. We look forward to its application in the precision Møller polarimetry at high energies.

\section{ACKNOWLEDGMENTS}

Useful discussions with Dr. M. Swartz and Dr. R. Prepost are acknowledged. We acknowledge the kind hospitality of Professor C. Prescott while this work was completed. This work was partly supported by the Polish Government KBN Grant No. 2P30225206 and by the U.S. Department of Energy Contracts DE-FG05-91ER40627 and DE-AC0376ER00515.
[1] See, for example, B. Pietryzk, in Proceedings of the Tennessee International Symposium on Radiative Corrections, edited by B. F. L. Ward (World Scientific, Singapore, 1995); A. Blondel, ibid.; S. Jadach et al., ibid.; E. A. Kuraev et al., ibid.; W. Hollik, ibid.; and references therein.

[2] See, for example, M. Swartz et al., Report No. SLAC-PUB6467, 1994 (unpublished); L. G. Levchuk, Nucl. Instrum. Methods A 345, 496 (1994); S. De Bievre, J. Math. Phys. 28, 2142 (1987); S. K. Choi et al., Phys. Rev. D 29, 1909 (1984); H. A. Olsen and Per Osland, ibid. 25, 2895 (1982).

[3] K. Abe et al., Phys. Rev. Lett. 73, 25 (1994).

[4] M. Swartz (private communication).

[5] D. R. Yennie, S. Frautschi and H. Suura, Ann. Phys. (N.Y.) 13,
379 (1961); see also K. T. Mahanthappa, Phys. Rev. 126, 329 (1961).

[6] S. Jadach and B. F. L. Ward, Comput. Phys. Commun. 56, 351 (1990).

[7] S. Jadach and B. F. L. Ward, Phys. Rev. D 40, 3582 (1989).

[8] S. Jadach, E. Ritchter-Wa̧s, B. F. L. Ward, and Z. Wạs, Comput. Phys. Commun. 70, 305 (1992).

[9] M. Böhm, A. Denner, and W. Hollik, Nucl. Phys. B 304, 687 (1988).

[10] E. A. Kuraev et al., Sov. J. Nucl. Phys. 32, 548 (1980).

[11] R. Gastmans and Y. Van Ham, Phys. Rev. D 10, 3629 (1974).

[12] L. L. DeRaad, Phys. Rev. D 11, 3328 (1975); L. L. DeRaad and Y. J. Ng, ibid. 11, 1586 (1975), and references therein. 
[13] B. F. L. Ward, Phys. Rev. D 36, 939 (1987); 42, 3249 (1990).

[14] F. A. Berends et al., Nucl. Phys. B 264, 243,265 (1986); 239, 382 (1984); 239, 395 (1984); 206, 61 (1982).

[15] Z. Xu, D.-H. Zhang, and L. Chang, Nucl. Phys. B 291, 392 (1987).

[16] R. Kleiss and W. J. Stirling, Nucl. Phys. B 262, 235 (1985).
[17] F. A. Berends, R. Kleiss, and W. Hollik, Nucl. Phys. B 304, 712 (1988).

[18] R. Kleiss, Z. Phys. C 33, 433 (1987).

[19] S. Jadach, E. Richter-Was, B. F. L. Ward, and Z. Was, Phys. Rev. D 44, 2669 (1991). 